\title{
Etiloterapia en el servicio de urgencias. Una revisión de la literatura
}

\author{
Ethylotherapy in the Emergency Service. A Review of the Literature
}

Recepción: 30 Mayo 2019 | Aceptación: 27 Septiembre 2019

\author{
Hernando Andrés Olaya Acosta \\ Médico especialista en Toxicología y Farmacología \\ Clínica. Profesor asociado, Facultad de Medicina, \\ Pontificia Universidad Javeriana-Hospital Universitario \\ San Ignacio, Bogotá, Colombia \\ Rafael Gustavo Castellanos Garzón ${ }^{a}$ \\ Médico especialista en Medicina de Urgencias, \\ Pontificia Universidad Javeriana. Médico \\ emergenciólogo, Hospital Universitario San Ignacio, \\ Bogotá, Colombia \\ Alberto Adolfo Vides Velásquez \\ Médico especialista en Medicina de Emergencias, \\ Universidad del Rosario, Bogotá, Colombia. Médico \\ emergenciólogo, Hospital Universitario San Ignacio, \\ Bogotá, Colombia \\ Catalina Rodríguez Prada \\ Médica residente de Medicina de Urgencias, Facultad \\ de Medicina, Pontificia Universidad Javeriana, Bogotá, \\ Colombia
}

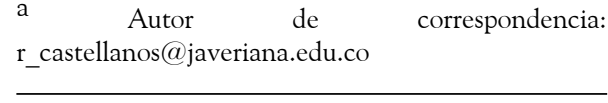

Cómo citar:: Olaya Acosta HA, Castellanos Garzón RG, Vides Velásquez AA, Rodríguez Prada C. Etiloterapia en el servicio de urgencias: una revisión de la literatura. Univ. Med. 2020;61(2). https://doi.or g/10.11144/Javeriana.umed61-2.etil.

\section{RESUMEN}

Desde hace décadas, el alcohol etílico ha sido utilizado como tratamiento de condiciones clínicas de origen tóxico, porque modifica procesos metabólicos que disminuyen los efectos deletéreos de algunos alcoholes tóxicos que, posterior a su exposición, generan un impacto orgánico importante e incluso la muerte. El fomepizol es el antídoto para los alcoholes tóxicos, y su mecanismo de acción es la inhibición de la alcohol deshidrogenasa. El etanol se utiliza terapéuticamente para inhibir la alcohol deshidrogenasa cuando el fomepizol no está disponible. Por esto, en este artículo se revisa la evidencia científica que hay con respecto al uso del alcohol etílico como terapia en la intoxicación con alcoholes, así como aspectos importantes del fomepizol.

Palabras clave

intoxicación; alcoholes tóxicos; etanol; metanol; etilenglicol; etiloterapia.

\section{ABSTRACT}

Ethyl alcohol has been used as a treatment for clinical conditions of toxic origin for decades, for the modification of metabolic processes that diminish the deleterious effects of some toxic alcohols that, after exposure, have an important organic impact and even death. Fomepizole is the antidote for toxic alcohols, and its mechanism of action is the inhibition of alcohol dehydrogenase. Ethanol is used therapeutically to inhibit alcohol 
Hernando Andrés Olaya Acosta, Rafael Gustavo Castellanos Garzón, Alberto Adolfo Vides Velásquez, ET AL.

dehydrogenase when fomepizole is not available. This is why in this article we want to evaluate the scientific evidence regarding the use of ethyl alcohol as a therapy in alcohol poisoning, as well as important aspects of fomepizole.

Keywords

alcoholic intoxication; ethanol; methanol; ethylene glycol.

\section{Introducción}

El alcohol etílico o etanol es un líquido incoloro de olor agradable que habitualmente se usa con fines recreativos y se considera una sustancia psicoactiva aceptada en distintos ambientes sociales, religiosos, culturales y legales en todo el mundo (1). Diversas bebidas contienen diferentes grados de alcohol etílico con rangos de entre el $4 \%$ y el $60 \%$ (2). Adicionalmente, existen preparados para su aplicación intravenosa que se utilizan con fines terapéuticos y concentraciones cercanas al 100 $\%$.

El etanol es un compuesto químico inflamable y de fórmula química $\mathrm{C}_{2} \mathrm{H}_{5} \mathrm{OH}$, sabor etéreo y soluble en el agua. Se obtiene, principalmente, a partir de dos métodos: la fermentación de azúcares y la síntesis a partir del etileno. Se usa en ámbitos como la medicina, la industria de fabricación de cosméticos, las bebidas y los perfumes (3).

El uso reconocido en medicina en labores de antisepsia ha mostrado eficacia similar a otros antisépticos (4); sin embargo, existen otras aplicaciones más allá de esta, objeto de la atención en los servicios de urgencias (tabla 1). $\mathrm{Su}$ uso con fines terapéuticos suele reconocerse como etiloterapia.

Tabla 1.

Usos del alcohol etílico en medicina

\begin{tabular}{|l|l|}
\hline Usos habituales & $\begin{array}{l}\text { Intoxicación por alcohol metílico } \\
\text { Intoxicación por dietilenglicol-etilenglicol } \\
\text { Intoxicación por fluoroacetato de sodio } \\
\text { Síndrome de abstinencia al alcohol }\end{array}$ \\
\hline $\begin{array}{l}\text { Usos experimentales o o } \\
\text { no relacionados con } \\
\text { atención de } \\
\text { intoxicaciones }\end{array}$ & $\begin{array}{l}\text { Hipertensión en el embarazo (5) } \\
\text { Diabetes tipo } 2^{*}(6)\end{array}$ \\
$\begin{array}{l}\text { Control de las concentraciones de colesterol en la sangre (7) } \\
\text { Mejoría de la motilidad intestinal (8) } \\
\text { Reducción del riesgo cardiovascular (9) } \\
\text { Reducción del riesgo de desarrollar demencias** (10) }\end{array}$ \\
\hline
\end{tabular}

*Dependiente del estilo de vida.

**Aplica con el consumo moderado de vino.
En el servicio de urgencias, la etiloterapia ha sido útil durante muchos años y sigue siendo empleada a pesar del desarrollo de inhibidores competitivos como el fomepizol, y de los cuales se describen algunas de las características principales (tabla 2). El objetivo de esta revisión es describir el uso de la etiloterapia en el servicio de urgencias, así como aspectos importantes del fomepizol.

\section{Tabla 2.}

Ventajas y desventajas del etanol y fomepizol

\begin{tabular}{|c|c|c|}
\hline & Alcohol etílico & Fomepizol \\
\hline Ventajas & $\begin{array}{l}\text { - Bajo costo } \\
\text { - Pacilidad para encontrarlo } \\
\text { ambientes de bajos recursos } \\
\text { para la atención } \\
\text { - Mayor experiencia clinica }\end{array}$ & $\begin{array}{l}\text { Mayor afinidad por la enzima } \\
\text { (alcohol deshidrogenasa) } \\
\text { - Menor proporción de eventos } \\
\text { adversos } \\
\text { - No requiere monitoreo } \\
\text { continuo de sus niveles } \\
\text { - No requiere necesariamente } \\
\text { hospitalización en cuidados } \\
\text { intensivos }\end{array}$ \\
\hline Desventajas & $\begin{array}{l}\text { Menor afinidad por la } \\
\text { enzima que el fomepizol } \\
\text { - Requiere monitoreo en } \\
\text { sangre de sus niveles } \\
\text { - Requiere hospitalización en } \\
\text { la unidad de cuidados } \\
\text { intensivos } \\
\text { - Mayor proporción de } \\
\text { eventos adversos }\end{array}$ & $\begin{array}{l}\text { - No disponible en todos los } \\
\text { centros médicos } \\
\text { - Mayor costo } \\
\text { - Menor experiencia con su uso }\end{array}$ \\
\hline
\end{tabular}

\section{Aspectos de la farmacocinética y farmacodinámica del alcohol etílico}

El etanol por vía oral se absorbe principalmente en el intestino delgado y su concentración es directamente proporcional a la concentración de alcohol presente en la bebida. Las concentraciones superiores al $40 \%$ no muestran correlación y, al contrario, ocurre el enlentecimiento de la absorción derivada de una menor velocidad de vaciamiento gástrico (11). Esto explica el porqué de las altas concentraciones de etanol utilizadas en el tratamiento de las intoxicaciones.

$\mathrm{Al}$ ser absorbido, se distribuye uniformemente en los tejidos, con máxima concentración en los tejidos de alto contenido de grasa (volumen de distribución [VD]: 0,57). La eliminación sin transformación es de aproximadamente el $2 \%$ a través de la orina y los pulmones (12). La eliminación pulmonar del etanol tiene muy poco interés clínico; solo es relevante en casos de pruebas judiciales. 
Su metabolismo principal es hepático, a través de tres enzimas: la alcohol deshidrogenasa, el sistema microsomal de oxidación (citocromo P450 2E1) y la catalasa (13). La vía metabólica a través de la alcohol deshidrogenasa es importante, en términos de explicar su utilidad en la intoxicación por otros alcoholes tóxicos, teniendo en cuenta que en estos casos actúa como inhibidor competitivo de la enzima y previene la formación de los metabolitos tóxicos. El alcohol es transformado de etanol a acetaldehído y, posteriormente, a acetato, el cual se puede utilizar, a su vez, para fabricar ácidos grasos o metabolizarlo mediante el ciclo de Krebs (14).

De forma aguda, el alcohol produce varios efectos fisiológicos (tabla 3). Ellos explican, al menos parcialmente, los eventos negativos que podrían presentarse durante la etiloterapia: confusión, estupor, somnolencia, depresión del estado de conciencia, hipotonía e hiperreflexia, náuseas y vómito, insuficiencia cardiaca, edema pulmonar, dificultad respiratoria, oliguria, dolor abdominal, necrosis tubular aguda, hematuria, proteinuria e insuficiencia renal aguda $(15,16)$.

\section{Tabla 3.}

Efectos agudos del etanol

\begin{tabular}{|l|l|}
\hline Sistema digestivo & $\begin{array}{l}\text { - Reducción de la presión basal, amplitud de sus } \\
\text { contracciones y la frecuencia de la relación del esfinter } \\
\text { esofágico inferior (17). } \\
\text { - Estimulación de la producción de gastrina y modificación } \\
\text { de la velocidad de secreción gástrica (18). } \\
\text { - Incremento de la motilidad y permeabilidad intestinal (19). }\end{array}$ \\
\hline Sistema cardiovascular & $\begin{array}{l}\text { - Reducción de la contractilidad cardiaca (20). } \\
\text { - Inducción de arritmias cardiacas (21). }\end{array}$ \\
\hline Sistema inmunológico & $\begin{array}{l}\text { - Incremento en la producción y liberación de citocinas } \\
\text { proinflamatorias (22). }\end{array}$ \\
\hline Sistema nervioso & $\begin{array}{l}\text { - Reducción de la velocidad de conducción entre las } \\
\text { neuronas (23). } \\
\text { - Reducción de la velocidad metabólica (24). } \\
\text { - Alteración del flujo sanguíneo cerebral (25). }\end{array}$ \\
\hline
\end{tabular}

\section{Uso en intoxicación por alcohol metílico- metanol}

El metanol es un líquido volátil e incoloro, pero sus metabolitos conducen a la toxicidad en el humano. La vía más frecuente de intoxicación es la oral, generalmente producto del consumo de bebidas alcohólicas adulteradas (26).

La toxicidad se genera como consecuencia de la oxidación del metanol a formaldehído y, posteriormente, a ácido fórmico (sustancia tóxica para el ser humano). La velocidad con la que se metaboliza el metanol, dependiente a su vez de la coingestión de etanol, modifica el tiempo de expresión sintomática de la intoxicación por metanol, la cual puede aparecer entre los 40 minutos y las 72 horas (27). Se ha documentado que la dosis mínima capaz de producir toxicidad es de $30 \mathrm{ml}$. La mortalidad de la intoxicación por alcohol metílico se encuentra entre el $10 \%$ y el $30 \%$ (28); además, se ha descrito que luego de la inhalación o exposición cutánea, estos dos últimos casos suelen ser un reto diagnóstico para el médico tratante (29).

El diagnóstico está basado en la sospecha clínica y los hallazgos en los exámenes de laboratorio (30); pero el diagnóstico de certeza se realiza mediante la detección y la cuantificación de las concentraciones plasmáticas (31). El tratamiento indicado incluye la administración de etanol o fomepizol, por su actividad inhibitoria sobre la alcohol deshidrogenasa, con el objeto de disminuir la producción del metabolito tóxico, ácido fólico y diálisis (32-34).

\section{Etanol}

La dosis de etanol utilizada varía según el antecedente o no de consumo prolongado de alcohol por parte del paciente (35). El uso combinado del etanol y la hemodiálisis se considera un método seguro y efectivo para controlar la intoxicación por metanol (36). Existen esquemas de manejo sugeridos para la etiloterapia que se presentan en la tabla 4. 
Hernando Andrés Olaya Acosta, Rafael Gustavo Castellanos Garzón, Alberto Adolfo Vides Velásquez, ET AL.

Tabla 4 .

Dosis de etanol en intoxicación por metanol

\begin{tabular}{|c|c|c|c|c|}
\hline \multicolumn{5}{|c|}{ Administración intravenosa } \\
\hline & \multicolumn{2}{|c|}{ Etanol al $5 \%$} & \multicolumn{2}{|c|}{ Etanol al $10 \%$} \\
\hline Dosis de carga & & 15 & & 7,5 \\
\hline Infusión* & & $2-4$ & & $1-2$ \\
\hline Infusión ${ }^{* *}$ & & $4-8$ & & $2-4$ \\
\hline Infusión con diálisis ${ }^{*}$ & & $4-7$ & & $2-3,5$ \\
\hline Infusión con diálisis ${ }^{* *}$ & & $6-10$ & & $3-5$ \\
\hline \multicolumn{5}{|c|}{ Administración oral } \\
\hline & $5 \%$ & $10 \%$ & $20 \%$ & $40 \%$ \\
\hline Dosis de carga & 15 & 7,5 & 4 & 2 \\
\hline Infusión* ${ }^{*}$ & 2 & 1 & 0,5 & 0,25 \\
\hline Infusión ${ }^{* *}$ & 4 & 2 & 1 & 0,5 \\
\hline Infusión con diálisis ${ }^{*}$ & 4 & 2 & 1 & 0,5 \\
\hline Infusión con diálisis ${ }^{* *}$ & 8 & 4 & 2 & 1 \\
\hline
\end{tabular}

Los datos se expresan en $\mathrm{ml} /$ $\mathrm{kg} / \mathrm{h}$. Fuente: Basada en (37). *No consumidor regular de bebidas alcohólicas. **Consumidor regular de bebidas alcohólicas.

La dosis de carga del etanol se encuentra entre los 600 y los $800 \mathrm{mg} / \mathrm{kg}$ y la dosis de mantenimiento es de $110 \mathrm{mg} / \mathrm{kg} / \mathrm{h}$. Cuando se posee alcohol absoluto (96\%), es posible diluir $50 \mathrm{ml}$ de este en $450 \mathrm{ml}$ de dextrosa en agua destilada para obtener una concentración del $10 \%$. Cuando no se dispone de este, se puede utilizar la siguiente fórmula para estimar el volumen de bebida alcohólica que debe suministrarse (38):

Volumen de alcohol $=$ Alcoholemia deseada $(\mathrm{mg} / \mathrm{dl}) \times$ volumen distribución $\times$ peso

$\%$ de la solución $\times$ gravedad específica $(0,79)$

Etanol. Las concentraciones de etanol deben ser superiores a $100 \mathrm{mg} / \mathrm{dl}$. Para lograrlo se utiliza una dosis de carga de $1 \mathrm{ml} / \mathrm{kg}$ diluidos en solución salina o dextrosa y, posteriormente, una infusión de $0,16 \mathrm{ml} / \mathrm{kg} / \mathrm{h}$. Su utilidad deriva de la competencia por la acción con la alcohol deshidrogenasa, que disminuye la formación de metabolitos tóxicos (37). Con el uso del etanol, la vida media del etilenglicol llega a ser de 17 h (con fomepizol es de $14 \mathrm{~h}$ ) (54). No existe evidencia científica de suficiente calidad que permita determinar con exactitud su eficacia contra el placebo o contra otro comparador.

\section{Fomepizol}

El fomepizol es un inhibidor de la enzima alcohol deshidrogenasa hepática (8000 veces más afín que el etanol y 80.000 veces más afín que el metanol) implicada en la transformación del alcohol metílico a ácido fórmico. Su mayor eficacia es en la administración temprana en la intoxicación, cuando los metabolitos activos aún no se han formado. El uso del fomepizol ha aumentado en los últimos años (mientras disminuye el del etanol), en buena medida por su mejor perfil de seguridad. El pronóstico y desenlaces logrados luego de su uso son dependientes del tiempo en el cual se haya administrado. El esquema de administración en los casos en los cuales no se utiliza la diálisis inicia con una carga de $15 \mathrm{mg} / \mathrm{kg}$ luego $10 \mathrm{mg} / \mathrm{kg}$ cada $12 \mathrm{~h}$ y después de las $48 \mathrm{~h}$ de $15 \mathrm{mg} / \mathrm{kg}$ cada 12 h $(39,40)$. Recientemente, un estudio de cohorte encontró que el uso de fomepizol se relaciona con una menor proporción de eventos adversos que el etanol (Hazard Ratio [HR] = 0,16; IC $95 \%$ : 0,06-0,40), la proporción de eventos severos fue también menor ( $5 \%$ en fomepizol vs. $20 \%$ en etanol) con una media de horas similar para el inicio (3 h) (41).

No es claro si el fomepizol es superior al etanol $(42,43)$; pero hay tener en cuenta que el fomepizol está incluido en la lista de la Organización Mundial de la Salud de medicaciones esenciales (44).

El uso del fomepizol ofrece dos ventajas sobre el etanol: menor carga de trabajo del equipo de salud, sin monitoreo sanguíneo del etanol (45). Estudios futuros deberán evaluar si desde el punto de vista de la costoefectividad se trata de una intervención superior. Un estudio reciente en República Checa mostró que el tratamiento con fomepizol incrementa el costo del tratamiento hasta en tres veces, comparado con el etanol (46); por supuesto, deberán realizarse comparaciones en cuanto a costoefectividad para el escenario local para una mejor comprensión de estas conclusiones.

Fomepizol. El principio terapéutico de su uso es similar que el del etanol; sin embargo, el 
fomepizol tiene mayor afinidad por el etanol y no requiere mediciones continuas para reajustar las dosis terapéuticas. Al igual que lo referido para la intoxicación con el metanol, el uso del fomepizol, comparado contra el etanol, se relaciona con una menor proporción de eventos adversos, aunque su eficacia es reportada como similar. Como ventajas del fomepizol se han documentado menores errores en la administración del tratamiento y la no necesidad de realizar monitoreo continuo; como desventaja se ha indicado la falta del tratamiento en algunos centros de salud (55). Suelen utilizarse como dosis de tratamiento (al igual que con la intoxicación por metanol) una dosis de carga de $15 \mathrm{mg} / \mathrm{kg}$, seguido por infusión durante al menos $36 \mathrm{~h}$ de $10 \mathrm{mg} / \mathrm{kg}(27)$.

\section{Uso en intoxicación por dietilenglicol- etilenglicol}

El dietilenglicol y el etilenglicol son similares al alcohol etílico, pero se diferencian por los grupos carboxilo presentes en su molécula. Se trata de líquidos inodoros, incoloros y volátiles, y principalmente se usan como solventes o anticongelantes en la industria; son tóxicos para el humano. Una vez consumidos, se produce una rápida absorción gástrica $(<1$ h), tiene vida media de $3,5 \mathrm{~h}$ y su principal ruta de eliminación es a través de la orina (<90\%) (47). En la mayoría de los casos, su consumo se da como consecuencia de un intento de suicidio, drogadicción, consumo de bebidas alcohólicas adulteradas o contaminación accidental. Se reconoce que son mal absorbidos por vía oral, por lo cual se requieren consumo relativamente importantes para que se produzca una intoxicación severa (48). La dosis tóxica del etilenglicol es de $1,5 \mathrm{ml} / \mathrm{kg}$ de peso y del dietilenglicol es de $0,17 \mathrm{mg} / \mathrm{kg}$ de peso. Otros glicoles como el trietilenglicol y tetraetilenglicol muestran un perfil clínico y toxicológico similar $(49,50)$.

Estos compuestos son metabolizados por la alcohol deshidrogenasa, que conduce a la formación del ácido glicólico y ácido oxálico (51).
El metabolismo y excreción de los metabolitos altera la función hepática y produce falla renal. Esta intoxicación se caracteriza por tres fases clínicas: neurológica $(0,5-12 \mathrm{~h}$ luego de la ingesta), cardiopulmonar (12-36 h) y renal $(24-72 \mathrm{~h})(52,53)$. Como manifestaciones clínicas, en estos casos es detectable el desarrollo de euforia, náuseas y vómito; posteriormente, sobreviene la depresión del sistema nervioso central, confusión, alucinaciones, temblores y tetania, también se ha descrito dolor abdominal y hematemesis. Posteriormente, se presentan síntomas y hallazgos paraclínicos respiratorios, cardiovasculares y renales asociados con la acción de los metabolitos tóxicos (30).

Dentro de los estudios paraclínicos es necesaria la evaluación de electrolitos, gases arteriales, pruebas de función hepática y renal y monitoreo de la función cardiovascular (49). Dentro del tratamiento se utiliza la etiloterapia o el fomepizol, piridoxina, ácido fólico y tiamina, por los requerimientos del metabolismo de estos compuestos. La terapia con etanol o fomepizol se utiliza en casos en los cuales se documentan niveles mayores a $20 \mathrm{mg} / \mathrm{dl}$ o en los cuales exista historia de consumo con identificación de acidosis.

\section{Uso en intoxicación por fluoroacetato de sodio}

El fluoroacetato de sodio, altamente tóxico, está prohibido en muchos países del mundo, pero ocasionalmente es utilizado como componente de venenos para ratas (56). Se ha documentado que el fluoroacetato de sodio impide pasos metabólicos del ciclo de Krebs que implican la función de la enzima aconitasa y que conducen a la acumulación del citrato y ácido láctico, que alteran la función mitocondrial (57). Poco se conoce sobre su toxicocinética: el pico de concentración podría darse entre 0,5 y 2,5 h luego de su ingestión, pero se desconoce la dosis tóxica para exposiciones por vía respiratoria (58). La acumulación de los metabolitos tóxicos suele ocurrir en el corazón y el sistema nervioso. Desde el punto de vista clínico, en las primeras $6 \mathrm{~h}$ 
Hernando Andrés Olaya Acosta, Rafael Gustavo Castellanos Garzón, Alberto Adolfo Vides Velásquez, ET AL.

de la intoxicación es posible identificar sialorrea, náuseas, emesis, ansiedad y agitación. Luego sobrevienen agitación, temblores, hipotermia y acidosis láctica.

El tratamiento incluye gluconato de calcio, monoacetato de glicerilo y alcohol etílico a dosis de la intoxicación por metanol o etilenglicol. En estos casos, el etanol es utilizado como fuente de energía celular alternativa, teniendo en cuenta que el ciclo de Krebs se encuentra bloqueado por el agente tóxico; sin embargo, poco se conoce sobre su eficacia clínica medida a través de investigaciones objetivas (59).

\section{Conclusión}

Las intoxicaciones por algunos agentes, como el metanol, el etilenglicol y el fluoroacetato de sodio, se presentan con alguna frecuencia en los servicios de urgencias. Históricamente, se ha utilizado el alcohol etílico como tratamiento para ellas, por sus efectos sobre los agentes tóxicos o el funcionamiento metabólico celular. Hoy en día, existe el fomepizol como antídoto, pues su uso está relacionado con una menor proporción de eventos adversos aunque su costo es mayor y su disponibilidad es menor. En la actualidad, los centros de referencia han migrado a este tipo de tratamiento, por la menor necesidad de monitorización y proporción de eventos adversos; no obstante, los centros de atención en los cuales no se disponga de la tecnología tienen en el alcohol etílico una herramienta terapéutica útil y eficaz.

\section{Referencias}

1. Babor T, Caetano R, Casswell $\mathrm{S}$, et al. El alcohol: un producto de consumo no ordinario [internet]. 2a ed. Washington: Organización Panamericana de la Salud; 2010. Disponible en: http://iris.paho.org/xml ui/handle/123456789/2836

2. Téllez Mosquera J, Cote Menéndez M. Alcohol etílico: un tóxico de alto riesgo para la salud humana socialmente aceptado. Rev Fac Med. 2006;54(1):32-47.

3. Acofarma. Alcohol etílico: ficha de información técnica [internet]. 2017. Disponible en: http://www.acofarma.com/admin/u ploads/descarga/4565-e30651ba1b931 8abd1dbed96c1a111461928eda1/main /files/Alcohol_et_lico.pdf

4. Charles D, Heal CF, Delpachitra M, Wohlfahrt M, Kimber D, Sullivan $\mathrm{J}$, et al. Alcoholic versus aqueous chlorhexidine for skin antisepsis: the Avalanche trial. CMAJ. 2017 Aug 8;189(31):E1008-16.

5. Alexander D, Northcross A, Wilson N, Dutta A, Pandya R, Ibigbami T, et al. Randomized controlled ethanol cookstove intervention and blood pressure in pregnant Nigerian women. Am J Respir Crit Care Med. 2017 Jun;195(12):1629-39.

6. Gepner Y, Golan R, HarmanBoehm I, Henkin Y, Schwarzfuchs D, Shelef I, et al. Effects of initiating moderate alcohol intake on cardiometabolic risk in adults with type 2 diabetes: a 2-year randomized, controlled trial. Ann Intern Med. 2015 Oct 20;163(8):569-79.

7. Vu KN Hoogeveen RC, Nambi V, Volcik KA, Boerwinkle E, et al BCM. Causal role of alcohol consumption in an improved lipid profile: The Atherosclerosis Risk in Communities (ARIC) Study. PLoS One. 2016;11(2):e0148765.

8. Heinrich H, Goetze O, Menne $\mathrm{D}$, Iten PX, Fruehauf $\mathrm{H}$, Vavricka $\mathrm{SR}$, et al. Effect on gastric function and symptoms of drinking wine, black tea, or schnapps with a Swiss cheese fondue: randomised controlled crossover trial. Br Med J. 2010 Dec 14;341:c6731. 
9. Bell S, Daskalopoulou M, Rapsomaniki E, George J, Britton A, Bobak M, et al. Association between clinically recorded alcohol consumption and initial presentation of 12 cardiovascular diseases: population based cohort study using linked health records. Br Med J. 2017 Mar;356:j909.

10. Von Arnim CA, Herbolsheimer F, Nikolaus T, Peter R, Biesalski HK, Ludolph AC, et al. Dietary antioxidants and dementia in a population-based case-control study among older people in South Germany. J Alzheimers Dis. 2012;31(4):717-24.

11. Wilkinson P. Pharmacokinetics of ethanol: a review. Alcohol Clin Exp Res. 1980;4(1):6-21.

12. Pizon AF, Becker CE, Bikin D. The clinical significance of variations in ethanol toxicokinetics. J Med Toxicol. 2007 Jun;3(2):63-72.

13. Paton A. Alcohol in the body. $\mathrm{Br}$ Med J. 2005;330(7482):85-7.

14. Lieber CS. Metabolism of alcohol. Clin Liver Dis. 2005 Feb;9(1):1-35.

15. Harrison NL, Skelly MJ, Grosserode EK, Lowes DC, Zeric T, Phister S, et al. Effects of acute alcohol on excitability in the CNS. Neuropharmacology. 2017 Aug 1;122:36-45.

16. Bjork JM, Gilman JM. The effects of acute alcohol administration on the human brain: insights from neuroimaging. Neuropharmacology. 2014 Sep;84:101-10.

17. Ferdinandis TG, Dissanayake AS, de Silva HJ. Chronic alcoholism and esophageal motor activity: a 24-h ambulatory manometry study. J Gastroenterol Hepatol. 2006 Jul;21(7):1157-62.

18. Stermer E. Alcohol consumption and the gastrointestinal tract. Isr Med Assoc J. 2002 Mar;4(3):200-2.
19. Davis 4th BT, Voigt RM, Shaikh M, Forsyth CB, Keshavarzian A. CREB Protein mediates alcoholinduced circadian disruption and intestinal permeability. Alcohol Clin Exp Res. 2017 Dec;41(12):2007-14.

20. Walker RK, Cousins VM, Umoh NA, Jeffress MA, Taghipour D, AlRubaiee M, et al. The good, the bad, and the ugly with alcohol use and abuse on the heart. Alcohol Clin Exp Res. 2013 Aug;37 (8):1253-60.

21. Sengul C, Cevik C, Ozveren O, Sunbul A, Oduncu V, Akgun T, et al. Acute alcohol consumption is associated with increased interatrial electromechanical delay in healthy men. Cardiol J. 2011;18(6):682-6.

22. Hu TM, Lee RP, Lee CJ, Subeq YM, Lin NT, Hsu BG. Heavy ethanol intoxication increases proinflammatory cytokines and aggravates hemorrhagic shock-induced organ damage in rats. Mediators Inflamm. 2013;2013:121786.

23. Valenzuela CF, Jotty $\mathrm{K}$. Mini-review: effects of ethanol on GABAA receptor-mediated neurotransmission in the cerebellar cortex--recent advances. Cerebellum. 2015 Aug;14(4):438-46.

24. Crews FT, Nixon K. Mechanisms of neurodegeneration and regeneration in alcoholism. Alcohol Alcohol. 2009;44(2):115-27.

25. Viski S, Orosz M, Czuriga-Kovacs KR, Magyar MT, Csiba L, Olah L. The acute effects of alcohol on cerebral hemodynamic changes induced by the head-up tilt test in healthy subjects. J Neurol Sci. 2016 Sep 15;368:113-20.

26. Grupo de Vigilancia y Control de Factores de Riesgo Ambiental del Instituto Nacional de Salud. Protocolo de vigilancia y control de intoxicaciones por 
Hernando Andrés Olaya Acosta, Rafael Gustavo Castellanos Garzón, Alberto Adolfo Vides Velásquez, ET AL.

metanol [internet]. 2010. Disponible en: https://www.minsalud.gov.co/com unicadosPrensa/Documents/INTOXI CACION_POR_METANOL.pdf. 27. Rietjens SJ, de Lange DW, Meulenbelt J. Ethylene glycol or methanol intoxication: which antidote should be used, fomepizole or ethanol? Neth J Med. 2014 Feb;72(2):73-9.

28. Rostrup M, Edwards JK, Abukalish M, Ezzabi M, Some D, Ritter H, et al. The methanol poisoning outbreaks in Libya 2013 and Kenya. PLoS One. 2016 Mar 31;11(3):e0152676.

29. Litovitz T. The alcohols: ethanol, methanol, isopropanol, ethylene glycol. Pediatr Clin North Am. 1986 Apr;33(2):311-23.

30. Kruse JA. Methanol and ethylene glycol intoxication. Crit Care Clin. 2012 Oct;28(4):661-711.

31. Jammalamadaka D, Raissi S. Ethylene glycol, methanol and isopropyl alcohol intoxication. Am J Med Sci. 2010 Mar;339(3):276-81.

32. Kapur BM, Vandenbroucke AC, Adamchik Y, Lehotay DC, Carlen PL. Formic acid, a novel metabolite of chronic ethanol abuse, causes neurotoxicity, which is prevented by folic acid. Alcohol Clin Exp Res. 2007 Dec;31(12):2114-20.

33. Kapur BM, Baber M. FASD: folic acid and formic acid - an unholy alliance in the alcohol abusing mother. Biochem Cell Biol. 2018 Apr;96(2):189-97.

34. Roberts DM, Yates C, Megarbane B, Winchester JF, Maclaren R, Gosselin $S$, et al. Recommendations for the role of extracorporeal treatments in the management of acute methanol poisoning: a systematic review and consensus statement. Crit Care Med. 2015 Feb;43(2):461-72.
35. McCoy HG, Cipolle RJ, Ehlers SM, Sawchuk RJ, Zaske DE. Severe methanol poisoning. Application of a pharmacokinetic model for ethanol therapy and hemodialysis. Am J Med. 1979 Nov;67 (5):804-7.

36. Ekins BR, Rollins DE, Duffy DP, Gregory MC. Standardized treatment of severe methanol poisoning with ethanol and hemodialysis. West J Med. 1985 Mar;142(3):337-40.

37. McMartin K, Jacobsen D, Hovda $\mathrm{KE}$. Antidotes for poisoning by alcohols that form toxic metabolites. Br J Clin Pharmacol. 2016 Mar;81(3):505-15.

38. Peña LM, Arroyave CL, Aristizábal JJ, Gómez UE. Toxicología clínica. Medellín: CIB; 2010. p. 322-33.

39. Sivilotti ML. Ethanol: tastes great! Fomepizole: less filling! Ann Emerg Med. 2009 Apr;53(4):451-3.

40. Brent J. Fomepizole for ethylene glycol and methanol poisoning. N Engl J Med. 2009 May 21;360(21):2216-23.

41. Lepik KJ, Levy AR, Sobolev BG, Purssell RA, DeWitt CR, Erhardt GD, et al. Adverse drug events associated with the antidotes for methanol and ethylene glycol poisoning: a comparison of ethanol and fomepizole. Ann Emerg Med. 2009 Apr;53(4):439-450.e10.

42. Beatty L, Green R, Magee $\mathrm{K}$, Zed P. A systematic review of ethanol and fomepizole use in toxic alcohol ingestions. Emerg Med Int. 2013;2013:638057.

43. Zakharov S, Pelclova D, Navratil T, Belacek J, Komarc M, Eddleston $\mathrm{M}$, et al. Fomepizole versus ethanol in the treatment of acute methanol poisoning: Comparison of clinical effectiveness in a mass poisoning outbreak. Clin Toxicol (Phila). 2015;53(8):797-806. 
44. World Health Organization. WHO model list of essential medicines [Internet]. 19th list. 2017. Disponible en: http://wwwwhoint/Medicines/Publi cations/Essentialmedicines/En

45. Zakharov S, Navratil T, Pelclova D. Fomepizole in the treatment of acute methanol poisonings: experience from the Czech mass methanol outbreak 2012. Biomed Pap Med Fac Univ Palacky Olomouc Czech Repub. 2014 Dec;158(4):641-9.

46. Rulisek J, Balik M, Polak F, Waldauf P, Pelclova D, Belohlavek J, et al. Cost-effectiveness of hospital treatment and outcomes of acute methanol poisoning during the Czech Republic mass poisoning outbreak. J Crit Care. 2017 Jun;39:190-8.

47. Snellings WM, McMartin KE, Banton MI, Reitman F, Klapacz J. Human health assessment for longterm oral ingestion of diethylene glycol. Regul Toxicol Pharmacol. 2017 Jun 30;87 Suppl 2:S1-20.

48. Schier JG, Barr DB, Li Z, Wolkin AF, Baker SE, Lewis LS, et al. Diethylene glycol in health products sold over-the-counter and imported from Asian countries. J Med Toxicol. 2011 Mar; 7 (1):33-8.

49. Gutiérrez de Salazar Medina MM. Dietilenglicol-etilenglicol. En: Guías para el manejo de urgencias toxicológicas. Bogotá: Ministerio de Salud de Colombia; 2008. p. 224-6.

50. Viinamaki J, Sajantila A, Ojanpera I. Ethylene glycol and metabolite concentrations in fatal ethylene glycol poisonings. J Anal Toxicol. 2015;39(6):481-5.

51. Herold DA, Keil K, Bruns DE. Oxidation of polyethylene glycols by alcohol dehydrogenase. Biochem Pharmacol. 1989 Jan 1;38(1):73-6.
52. Mintegi S. Manual de intoxicaciones en pediatría. 3a ed. Madrid: Sociedad Española de Urgencias de Pediatría; 2012.

53. Gopalakrishnan N, Kamarajan M, Balasubramaniyan T, Sakthirajan R, Dhanapriya J, Dineshkumar T. Diethylene glycol poisoning-induced acute kidney injury. Saudi J Kidney Dis Transpl. 2016;27(6):1276-9.

54. Holstege CP, Rusyniak DE. Medical toxicology. Med Clin North Am. 2005;89(6).

55. Thanacoody RH, Gilfillan C, Bradberry SM, Davies J, Jackson G, Vale AJ, et al. Management of poisoning with ethylene glycol and methanol in the UK: a prospective study conducted by the National Poisons Information Service (NPIS). Clin Toxicol (Phila). 2016;54(2):134-40.

56. Kalmbach ER. "Ten-Eighty" a WarProduced Rodenticide. Science. 1945 Aug 31;102 (2644):232-3.

57. Proudfoot AT, Bradberry SM, Vale JA. Sodium fluoroacetate poisoning. Toxicol Rev. 2006;25(4):213-9.

58. Eason Miller, A, Ogilvie, E, Fairweather A CT. An updated review of the toxicology and ecotoxicology of sodium fluoroacetate (1080) in relation to its use as a pest control tool in New Zealand. N Z J Ecol. 2011;35(1):1-20.

59. Granada J, Rodríguez D. Intoxicación por fluoroacetato de sodio: reporte de caso. Rev Fac Med Univ Nac Colomb. 2014;62(1):137-40.

\section{Notas}

Conflictos de interés: los autores no reportan conflictos de interés. 